\title{
Dengue: descrevendo a epidemia em Aracaju, Sergipe, Brasil, 2008
}

\author{
Dengue: describing the epidemic in Aracaju, Sergipe, Brazil, 2008 \\ Dengue: la descripción de la epidemia en Aracaju, Sergipe, Brasil, 2008 \\ Paulo Emidio Lobão Cunha ${ }^{1 *}$, Anna Klara Bohland²
}

Palavras-chave:

Dengue

Epidemiologia

Vigilância Epidemiológica

\author{
Keywords: \\ Dengue \\ Epidemiology \\ Epidemiological Surveillance
}

\section{Resumo}

Introdução: a dengue é um dos maiores problemas de saúde pública no Brasil, caracterizando-se como doença febril aguda, com espectro clínico variando desde quadros febris inespecíficos até manifestações graves com hemorragia e choque. A Vigilância Epidemiológica (VE) da dengue deve ter agilidade suficiente para detectar precocemente as epidemias, e juntamente com a organização da rede de assistência, minimizar os casos de evolução grave, reduzindo a letalidade. Objetivos: estudar o perfil epidemiológico da dengue na cidade de Aracaju no ano de 2008. Métodos: foi realizado um estudo transversal, sendo utilizadas as seguintes variáveis: sexo, idade do paciente, cor da pele, semana epidemiológica de notificação, região de saúde, classificação da forma clínica da doença, resultado do exame (Mac-Elisa) e óbito. As informações foram colhidas do banco de dados do Sistema de Informações de Agravos de Notificação da Secretaria de Estado da Saúde de Sergipe. Os dados foram formatados utilizando o programa Microsoft Office Excel ${ }^{\circledR}$ e transferidos para o Stats Direct (versão 2.7.9), onde foi realizada a análise. Resultados: foram notificados 11348 casos e 10165 casos confirmados de dengue, com taxa de incidência de 1,89 casos/1000 habitantes em 2008. A maior incidência (por mil habitantes) foi sexo feminino (20,5), entre os 5 e 15 anos $(26,1)$ e cor de pele parda $(28,1)$. No que se refere à semana epidemiológica, o período compreendido entre a 9a e a $24^{\mathrm{a}}$ semanas apresentou 0 maior número de casos. A região de saúde com maior coeficiente foi a quarta $(22,3)$. Quanto à realização do exame, 43,1\% dos casos graves foram confirmados sem a sua realização. Dos casos confirmados, 96,1\% foram classificados como dengue clássica, 2,6\% como dengue com complicações, 1,2\% como febre hemorrágica da dengue e apenas 2 casos $(0,02 \%)$ como síndrome do choque da dengue. 0 diagnóstico final de dengue comparado à sorologia apresentou uma sensibilidade de 99,4\% e uma especificidade de 97,0\%. A taxa de letalidade nos casos graves foi de 4,6\%. Conclusão: 0 melhor entendimento epidemiológico da doença permitirá 0 aprimoramento da vigilância, detectando mais precocemente e evitando novas epidemias da doença.

\section{Abstract}

Introduction: Dengue is a major public health problem in Brazil, characterized as acute febrile illness with clinical spectrum ranging from unspecific febrile to severe manifestations such as bleeding and shock. The dengue epidemiological surveillance (ES) must be agile enough to detect early outbreaks and cases of severe outcome, reducing lethality. Objectives: To study the epidemiology of dengue in the city of Aracaju in 2008. Methods: We performed a cross-sectional study, which used the following variables: sex, patient age, race, epidemiological week of notification, health district, classification of the clinical form of the disease, serological test results (Mac-Elisa) and death. The information was collected from the database of the Information System for Notifiable Diseases of the Secretary of Health of Sergipe. The data were formatted in another database, using the Microsoft Office Excel ${ }^{\circledR}$, and later transferred to Stats Direct (version 2.7.9), which we performed a statistical analysis. Results: We reported 11348 suspected dengue cases and 10165 confirmed cases of dengue, assuming an incidence rate of 1.89 cases/1000 inhabitants in 2008. The incidence rate (cases/1000 inhabitants) were most affected females (20.5), 5 to 15 years (26.1) and brown skin color (28.1). With regard to epidemiological week, it was observed the period between the $9^{\text {th }}$ and $24^{\text {th }}$ week was the most epidemic. The most affected district was the fourth (22.3). As for the serological test, $43.1 \%$ of the

\footnotetext{
${ }^{1}$ Universidade Federal de Sergipe (UFS). paulinhoemidio@hotmail.com

${ }^{2}$ Universidade Federal de Sergipe (UFS). anna.bohland@uol.com.br

${ }^{*}$ Autor correspondente.

Fonte de financiamento: PIBIC/CNPq/UFS

Conflito de interesses: declaram não haver.

Recebido em: 14/09/2011

Aprovado em: 12/12/2012
} 
serious cases were confirmed without their realization. Of the confirmed cases, $96.1 \%$ were classified as dengue fever, $2.6 \%$ such as dengue fever with complications and $1.2 \%$ such as Dengue Hemorrhagic Fever and only 2 patients $(0.02 \%)$ with dengue shock syndrome. The final diagnosis of dengue compared to serology had had a sensitivity of $99.4 \%$ and a specificity of $97.0 \%$. The fatality rate in severe cases was $4.6 \%$. Conclusion: better understanding the epidemiology of the disease will allow the improvement of surveillance, detecting earlier and avoiding new epidemic disease.

\section{Palabras clave: \\ Dengue \\ Epidemiología \\ Vigilancia Epidemiológica}

\section{Resumen}

Introducción: El dengue es un problema importante de salud pública del Brasil, que si caracteriza como una enfermedad febril aguda con espectro clínico que van desde fiebre inespecífica a manifestaciones más graves como la hemorragia y el shock. La vigilancia epidemiológica del dengue debe ser lo suficientemente ágil para detectar los brotes y principios de los casos graves, y reducir la mortalidad. Objetivos: Estudiar la epidemiologia del dengue en la ciudad de Aracaju, en 2008. Métodos: Se realizó un estudio transversal, que utiliza las siguientes variables: sexo, edad del paciente, color de la piel, la semana epidemiológica de declaración, el distrito sanitario, clasificación de la forma clínica de la enfermedad, los resultados de serología (Mac-Elisa) y la muerte. La información se obtuvo a partir de la base de datos del Sistema de Información de Enfermedades de Declaración Obligatoria de la Secretaría de Estado de Salud de Sergipe. Los datos se formatearon en otra base de datos, con el uso de Microsoft Office Excel $^{\circledR}$, y más tarde transferida al software Stats Direct (version 2.7.9) que se realizó un análisis estadístico. Resultados: Se registraron 11348 casos sospechosos e 10165 casos confirmados del dengue, asumiendo una tasa de incidencia de 1,89 casos/1.000 habitantes en 2008. Las tasas más altas de incidencia fueron mujeres $(20,5)$, edad entre 5 y 15 años $(26,1)$ y piel de color pardo $(28,1)$. Con respecto a la semana epidemiológica, se observó el período comprendido entre el $9^{\circ}$ y $24^{\circ}$ semana con el mayor número de casos. El distrito sanitario con mayor coeficiente fue el cuarto $(22,3)$. En cuanto a la serología, 43,2\% de los casos más graves se han confirmado sin su realización. De los casos confirmados, 96,1\% fueron clasificados como dengue, $2,6 \%$ como dengue con complicaciones y 1,2\% como el dengue hemorrágico. Apenas 2 casos (0,02\%) como síndrome de choque del dengue. El diagnóstico final en comparación con la serología tiene una sensibilidad del 99,4\% y una especificidad del 97,0\%. La tasa de mortalidad en los casos graves fue del 4,6\%. Conclusión: una mejor comprensión de la epidemiología de la enfermedad permitirá la mejora de la vigilancia, la detección temprana y evitar nuevos brotes.

\section{Introdução}

A dengue é a arbovirose de maior importância ${ }^{1}$ e de maior incidência no mundo ${ }^{2}$. Aproximadamente 1,3 bilhóes de pessoas estão em risco constante de infecção ${ }^{3}$, principalmente em países tropicais, cuja temperatura e umidade favorecem a proliferaçáo do mosquito vetor, pertencente aos culicídeos do gênero Aedes ${ }^{4}$.

A doença é caracterizada como febril aguda, com espectro clínico variando desde quadros febris inespecíficos até manifestações graves com hemorragia e choque: a febre hemorrágica da dengue (FHD) e a síndrome do choque da dengue $(\mathrm{SCD})^{5}$. A doença é causada por um Flavivirus, com quatro sorotipos conhecidos. Estima-se que no mundo, anualmente, entre 50 e 100 milhōes de pessoas são infectadas pelo vírus, dos quais aproximadamente 500 mil apresentam dengue hemorrágica e cerca de 12 mil vão a óbito ${ }^{6,7}$.

Em 2008, foram notificados 585.769 casos no Brasil, marcando o pior cenário da doença no país em relação ao total de internaçôes e óbitos ${ }^{8}$. Destes casos, 20.116 (3,4\%) foram confirmados como dengue com complicaçōes (DCC), com 302 óbitos, e 4.455 (0,8\%) foram confirmados como FHD, com 259 óbitos. Comparando-se esses dados com os registrados em 2007, houve aumento de 93,4\% no número total de óbitos 9

Como grande recurso para o controle da dengue, tem-se a vigilância epidemiológica (VE). Ela serve de base para reunir informaçóes, processar, analisar e interpretar os dados, planejar e adotar medidas de controle imediatas ou de médio e longo prazo $^{10}$. A VE da dengue deve ter agilidade suficiente para detectar precocemente as epidemias e casos de evolução grave, reduzindo a letalidade ${ }^{1}$.

O Sistema de Informação de Agravos de Notificação (SINAN) foi criado em 1990, pelo Ministério da Saúde, com o objetivo de coletar e processar dados sobre agravos de notificação em todo o território nacional, para fazer uma avaliação epidemiológica da doença e desencadear ações de controle, permitindo análise do comportamento epidemiológico ${ }^{9}$. A validade do sistema depende diretamente da qualidade dos dados, influenciada pela proporção de fichas de notificação preenchidas adequadamente e pelo cuidado no seu processamento ${ }^{11}$. A má qualidade dos dados gera distorçôes quanto a sua interpretação e, consequentemente, as falhas nas políticas de controle e prevenção da dengue.

Diante desse quadro e conhecendo a complexidade e a extensão do problema da dengue, o objetivo deste trabalho é descrever a situaçáo epidemiológica da doença no ano de 2008, em Aracaju, Sergipe, marcado por uma das maiores epidemias já existentes em Sergipe e no Brasil.

\section{Métodos}

Foi realizado um estudo transversal e retrospectivo, utilizando-se dados secundários referentes a todos os casos notificados de dengue dos residentes na cidade de Aracaju, 
no período de janeiro a dezembro de 2008. Como o estudo enfoca a situaçáo da dengue no município de Aracaju, faz-se necessário explicitar algumas das suas características: Aracaju é a capital do Estado de Sergipe, localiza-se no leste sergipano (latitude $10^{\circ} 55^{\prime} 56^{\prime \prime}$ e longitude $37^{\circ} 4^{\prime} 23^{\prime \prime}$ ), apresenta uma área terrestre de $181,8 \mathrm{~km}^{2}$ e altitude de 2 metros, a populaçáo estimada para o ano de 2008 pelo Instituto Brasileiro de Geografia e Estatística (IBGE) era de 536.785 habitantes $^{12}$.

Os dados do estudo foram obtidos a partir do banco de dados do Sistema de Informações de Agravos de Notificação (SINAN) $)^{13}$ da Secretaria de Estado da Saúde do Governo de Sergipe. O banco de dados era formado inicialmente por 11.761 casos notificados. Destes, 288 foram eliminados por apresentarem duplicidade, que teve como critério o nome do indivíduo, a data da notificação e o número da notificação. Posteriormente, 125 fichas foram eliminadas por se tratarem de casos notificados de outro município ou endereços onde não foi possível identificar o distrito sanitário ao qual pertenciam os casos. Após esta última eliminação, restaram 11.348 casos notificados da doença.

Utilizando o programa Microsoft Office Excel $^{\circledR}$, foi realizada a formatação dos dados e a exclusão das variáveis que não seriam utilizadas neste estudo. Por fim, este último banco de dados foi transferido para o software Stats Direct (versão 2.7.9) onde foi realizada a análise estatística. As variáveis estudadas foram: procedência da notificação, sexo, idade, cor da pele, semana epidemiológica, regiáo de residência, resultado do exame, classificação da forma clínica da doença (neste caso foram consideradas as notificaçóes de casos confirmados de dengue por laboratório ou por critérios clínico-epidemiológicos), dados pluviométricos e óbito confirmados pela doença.

Os resultados analisados receberam tratamento estatístico pertinente (média, desvio-padrão, freqüências absolutas, percentuais, taxas de incidência e letalidade da doença). Os dados pluviométricos foram coletados na Secretaria do Estado do Meio Ambiente e dos Recursos Hídricos ${ }^{14}$. Para o cálculo da populaçáo, foram utilizados os dados do IBGE $^{12}$. Também foi realizado o cálculo da sensibilidade e especificidade do diagnóstico final da Dengue, usando como padráo-ouro o exame sorológico Mac-Elisa. $\mathrm{O}$ teste exato de Fisher-Fremann-Halton foi o teste estatístico empregado para avaliar a influência das variáveis: sexo, idade, cor da pele, sorologia, semana epidemiológica e regiâo de saúde de residência com o coeficiente de incidência e de letalidade, admitindo-se um nível de significância estatística $\mathrm{p}<0,05$.

O estudo em questão foi aprovado pela Comissão Nacional de Ética em Pesquisa (CONEP) do Ministério da Saúde, sob o número de Certificado de Apresentação para Apreciação Ética (CAAE), protocolo 1929-0.000.107-09.

\section{Resultados}

De 11.348 novos casos notificados de dengue de residentes no município de Aracaju, foram confirmados 10.165 $(89,6 \%)$, resultando numa taxa de incidência de 18,9 casos por 1000 habitantes em 2008. Com relação à procedência das notificaçóes, aproximadamente metade dos casos (49,8\%) foram de hospitais localizados no município e os demais das 43 Unidades Básicas de Saúde.

Quanto ao sexo, 57,9\% eram do sexo feminino (Tabela 1). A média das idades dos indivíduos acometidos foi de 24,2 anos com desvio-padrão (DP) de 17,1 anos, observando maior predominância $(32,0 \%)$ em indivíduos de 15 a 30 anos. Foi observado também um grande número de crianças acometidas pela doença, pois a população com idades menores de 15 anos representou $34,5 \%$ do total de casos.

Tabela 1. Perfil demográfico, temporal e espacial dos casos confirmados de dengue e respectivo coeficiente de incidência, em Aracaju, Sergipe, 2008.

\begin{tabular}{|c|c|c|c|}
\hline Variáveis & Número & $\%$ & $\begin{array}{c}\text { C. de } \\
\text { Incidência }\end{array}$ \\
\hline \multicolumn{4}{|l|}{ Sexo } \\
\hline Masculino & 4284 & 42,1 & 17,2 \\
\hline Feminino & 5881 & 57,9 & 20,5 \\
\hline \multicolumn{4}{|l|}{ Idade (anos) } \\
\hline $0 \mid-5$ & 1113 & 11,0 & 25,5 \\
\hline $5 \mid-15$ & 2378 & 23,5 & 26,1 \\
\hline $15 \mid-30$ & 3238 & 32,0 & 20,7 \\
\hline $30 \mid-45$ & 2041 & 20,2 & 16,6 \\
\hline $45 \mid-60$ & 1003 & 9,9 & 12,9 \\
\hline 60 e mais & 356 & 3,5 & 7,9 \\
\hline \multicolumn{4}{|l|}{ Cor da pele } \\
\hline Branca & 1498 & 14,7 & 8,7 \\
\hline Negra & 468 & 4,6 & 8,2 \\
\hline Amarela & 56 & 0,6 & 7,6 \\
\hline Parda & 2855 & 28,1 & 9,6 \\
\hline Indígena & 28 & 0,3 & 12,6 \\
\hline Ignorado & 5260 & 51,7 & \\
\hline \multicolumn{4}{|c|}{ Semana epidemiológica } \\
\hline Semana 1 I-9 & 232 & 2,3 & 0,4 \\
\hline Semana 9 |-17 & 3709 & 36,5 & 6,9 \\
\hline Semana 17 |-25 & 5850 & 57,6 & 10,9 \\
\hline Semana 25 I-33 & 327 & 3,2 & 0,6 \\
\hline Semana $33 \mid-41$ & 23 & 0,2 & 0,0 \\
\hline Semana 41 I-53 & 24 & 0,2 & 0,0 \\
\hline \multicolumn{4}{|l|}{ Região de saúde } \\
\hline 1 & 2789 & 27,4 & 17,3 \\
\hline 2 & 2719 & 26,7 & 17,1 \\
\hline 3 & 2216 & 21,8 & 20,7 \\
\hline 4 & 2441 & 24,0 & 22,3 \\
\hline Total & 10165 & 100,0 & 18,9 \\
\hline
\end{tabular}

*excluídos 34 casos com idade ignorada. Fonte: Sergipe (2010) ${ }^{13}$. 
No tocante à análise da variável cor da pele, mais da metade das notificaçóes $(51,7 \%)$ apresentaram resultado ignorado. Observou-se que, somadas às fichas de notificação que apresentavam cor de pele parda e negra, estas tiveram maior frequência $(32,7 \%)$ que as demais (branca, amarela e indígena) com $15,6 \%$.

No que se refere à semana epidemiológica (Figura 1), foi observado uma média de 191,8 casos com variação de 0 a 1173 casos confirmados/semana (DP de 24,3 casos). De acordo com a Tabela 1, o número de casos foi relativamente baixo nas primeiras oito semanas (2,3\%). Nas oito semanas seguintes, houve um explosivo aumento em mais de 15 vezes do valor apresentado no mesmo período anterior, tendo posteriormente um aumento de $157,7 \%$ dos casos nas oito semanas seguintes, obtendo expressiva queda de casos a partir da $25^{\mathrm{a}}$ semana. Neste período, compreendido entre a $9^{\mathrm{a}}$ e a $24^{\mathrm{a}}$ semana, foram notificados $94,0 \%$ do total de casos de dengue em 2008, com pico máximo na $19^{a}$ semana $(11,5 \%)$. Observa-se na Figura 1 que o número de casos com sorologia positiva para dengue acompanhou a curva dos casos confirmados.

Dos casos confirmados como dengue, por critérios laboratoriais ou clínico-epidemiológicos (Tabela 2), 96,1\% apresentaram classificação final de dengue clássico (DC); 2,6\% como dengue clássico com complicaçôes (DCC); 1,2\% como febre hemorrágica de dengue (FHD); e 0,02\% apresentaram síndrome do choque de dengue (SCD).

No estudo da variável resultado do exame, observou-se a realização da sorologia em $15,4 \%$ do total de casos notificados. Destes, $61,3 \%$ foram confirmados laboratorialmente como

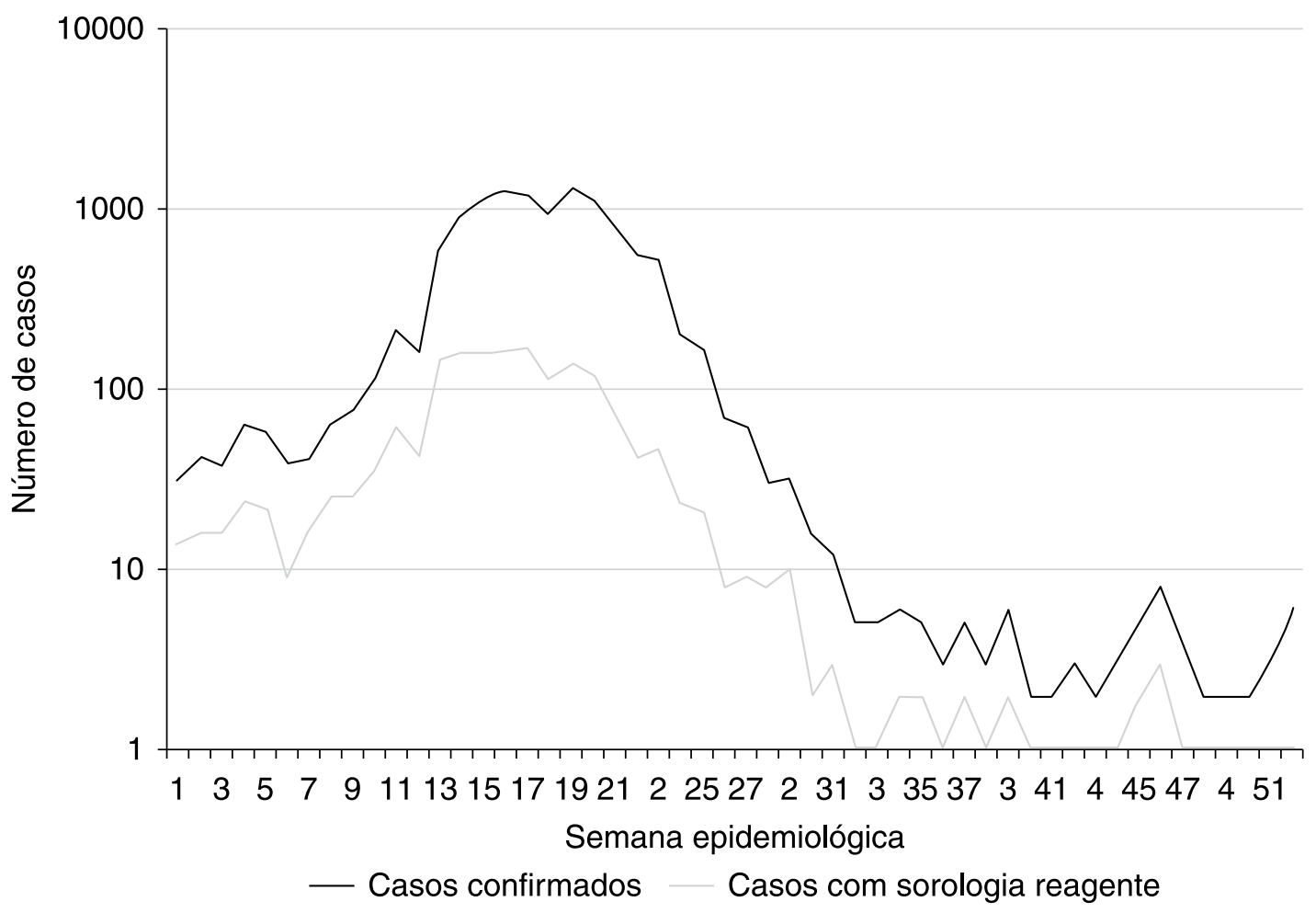

Figura 1. Casos de dengue confirmados e casos com sorologia positiva para dengue, segundo semana epidemiológica. Aracaju, Sergipe, 2008. Fonte: Sergipe (2010) ${ }^{13}$.

Tabela 2. Classificação final da forma clínica da Dengue de acordo com o critério de confirmação empregado para os casos confirmados de dengue em Aracaju, Sergipe, 2008.

\begin{tabular}{lcccc}
\hline \multirow{2}{*}{ Classificação final } & \multicolumn{2}{c}{ Critério } & Total & \\
\cline { 2 - 5 } & Laboratorial & Clínico-epidemiológico & Número & 9773 \\
\hline Dengue clássico & 1508 & 8265 & 268 & 96,1 \\
Dengue com complicações & 99 & 169 & 122 & 2,6 \\
Febre hemorrágica de dengue & 122 & - & 2 & 1,2 \\
Síndrome do choque de dengue & 2 & - & 10165 & 0,0 \\
Total & 1731 & 8434 & 100,0 \\
\hline
\end{tabular}

Fonte: Sergipe (2010) ${ }^{13}$. 
Tabela 3. Relação entre o diagnóstico de Dengue e o resultado do exame sorológico pelo Mac-Elisa em casos notificados da doença em Aracaju, Sergipe, 2008.

\begin{tabular}{|c|c|c|c|c|c|c|}
\hline \multirow{3}{*}{ Dengue } & \multicolumn{4}{|c|}{ Sorologia } & \multirow{2}{*}{\multicolumn{2}{|c|}{ Total }} \\
\hline & \multicolumn{2}{|c|}{ Sim } & \multicolumn{2}{|c|}{ Não } & & \\
\hline & Número & \%Percentual & Número & \%Percentual & Número & \%Percentual \\
\hline Sim & 1731 & 60.9 & 63 & 2.2 & 1794 & 63.1 \\
\hline Não & 11 & 0.4 & 1036 & 36.5 & 1047 & 36.9 \\
\hline Total & 1742 & 61.3 & 1099 & 38.7 & 2841 & 100.0 \\
\hline
\end{tabular}

Fonte: Sergipe (2010) ${ }^{13}$.

Tabela 4. Relação entre a presença de óbito com as variáveis demográficas e o resultado do exame sorológico Mac-ELISA dos casos confirmados de dengue em Aracaju, Sergipe, 2008.

\begin{tabular}{|c|c|c|c|c|}
\hline \multirow[t]{2}{*}{ Variáveis } & \multicolumn{2}{|c|}{ Óbito por dengue } & \multirow[t]{2}{*}{ Letalidade } & \multirow[t]{2}{*}{ Valor $\mathrm{p}$} \\
\hline & Sim & Não & & \\
\hline \multicolumn{5}{|l|}{ Sexo } \\
\hline Masculino & 10 & 4248 & 0,23 & \multirow{2}{*}{0,3394} \\
\hline Feminino & 8 & 5840 & 0,14 & \\
\hline \multicolumn{5}{|l|}{ Idade } \\
\hline $0 \mid-5$ & 4 & 1104 & 0,36 & \multirow{6}{*}{0,9876} \\
\hline $5 \mid-15$ & 1 & 2358 & 0,04 & \\
\hline $15 \mid-30$ & 3 & 3225 & 0,09 & \\
\hline $30 \mathrm{I}-45$ & 3 & 2026 & 0,15 & \\
\hline $45 \mid-60$ & 5 & 988 & 0,50 & \\
\hline 60 e mais & 2 & 352 & 0,56 & \\
\hline \multicolumn{5}{|c|}{ Negros ou pardos* } \\
\hline Sim & 13 & 3284 & 0,39 & \multirow{2}{*}{0,6053} \\
\hline Não & 4 & 1562 & 0,26 & \\
\hline \multicolumn{5}{|l|}{ Sorologia } \\
\hline Sim & 6 & 1708 & 0,35 & \multirow{2}{*}{0,1058} \\
\hline Não & 12 & 8333 & 0,14 & \\
\hline \multicolumn{5}{|l|}{ Região } \\
\hline 1 & 5 & 2770 & 0,18 & \multirow{5}{*}{0,9876} \\
\hline 2 & 4 & 2693 & 0,15 & \\
\hline 3 & 4 & 2199 & 0,18 & \\
\hline 4 & 5 & 2426 & 0,21 & \\
\hline Total & 18 & 10088 & 0,18 & \\
\hline
\end{tabular}

*excluídos os casos ignorados. Fonte: Sergipe (2010) ${ }^{13}$.

positivos. A relação entre a presença do diagnóstico de Dengue e o resultado apresentado pelo exame sorológico Mac-Elisa pode ser observada na Tabela 3. Inferiu-se, no presente estudo, uma sensibilidade de $99,4 \%$ e uma especificidade de $94,3 \%$ no diagnóstico final de dengue dos casos notificados nos quais consta a realizaçáo da sorologia.

A letalidade da doença foi de $0,2 \%$, apresentando-se maior na população idosa ( $\geq 60$ anos), atingindo o valor de $0,6 \%$. Porém, quando considerados somente os casos confirmados graves da doença (DCC, FHD e SCD), o coeficiente de letalidade subiu para $4,6 \%$, atingindo $10,0 \%$ para os pacientes com idade igual ou maior a 60 anos.

Analisando os casos de óbito (Tabela 4), os grupos de maior letalidade foram o masculino $(0,23 \%, \mathrm{p}=0,3394)$, negros e pardos $(0,39 \%, p=0,6053)$ e residentes na quarta regiâo de saúde, cuja letalidade foi $0,2 \%(\mathrm{p}=0,9876)$.

A onda epidêmica iniciou no final do mês de março de 2008, sugerindo que o aumento da circulação viral está relacionado ao aumento do índice pluviométrico (Figura 2).

\section{Discussão}

Neste estudo verificou-se uma incidência de 18,9 casos/1000 habitantes em 2008, considerada epidêmica por ser maior que 0,3 casos $/ 1.000$ habitantes ${ }^{10}$. Quanto à análise do preenchimento dos dados, estes apresentavam um amplo e satisfatório número de informaçóes, tais como o nome (que apesar de alguns erros de digitação, estava completo e presente em todas as fichas), o sexo e a semana de notificaçáo, também presentes em todas elas. No entanto, algumas falhas foram observadas com a variável idade, pois 36 fichas não apresentavam este dado ou os apresentavam como valor zero. Com a variável cor da pele, mais da metade das fichas constava como ignorado, apesar de todas elas apresentarem o dado preenchido.

$\mathrm{Na}$ análise da variável sexo, observou-se um predomínio do sexo feminino $(57,9 \%)$. Isso aconteceu provavelmente, por que as mulheres permaneceram mais tempo em suas residências do que os homens (e como a transmissão se faz principalmente no domicílio, a diferença observada pode justificar a maior exposição), ou por estas procurarem mais o serviço de saúde ${ }^{15}$. Barbosa et al. ${ }^{16}$ encontraram a distribuição semelhante entre os anos de 2000 e 2008, embora no ano de 2009 a proporção de casos em homens tenha superado a proporção em mulheres no Estado do Rio Grande do Norte.

Observando apenas os casos de óbito, os homens tiveram predomínio $(55,6 \%)$, apresentando as formas mais graves $\mathrm{da}$ doença ((DCC, FHD e SCD). Isso pode sugerir a hipótese de que os homens apresentam maior letalidade por procurarem menos os serviços de saúde do que as mulheres, porém, não houve significância estatística para o óbito com esta variável $(\mathrm{p}=0,3394)$. 


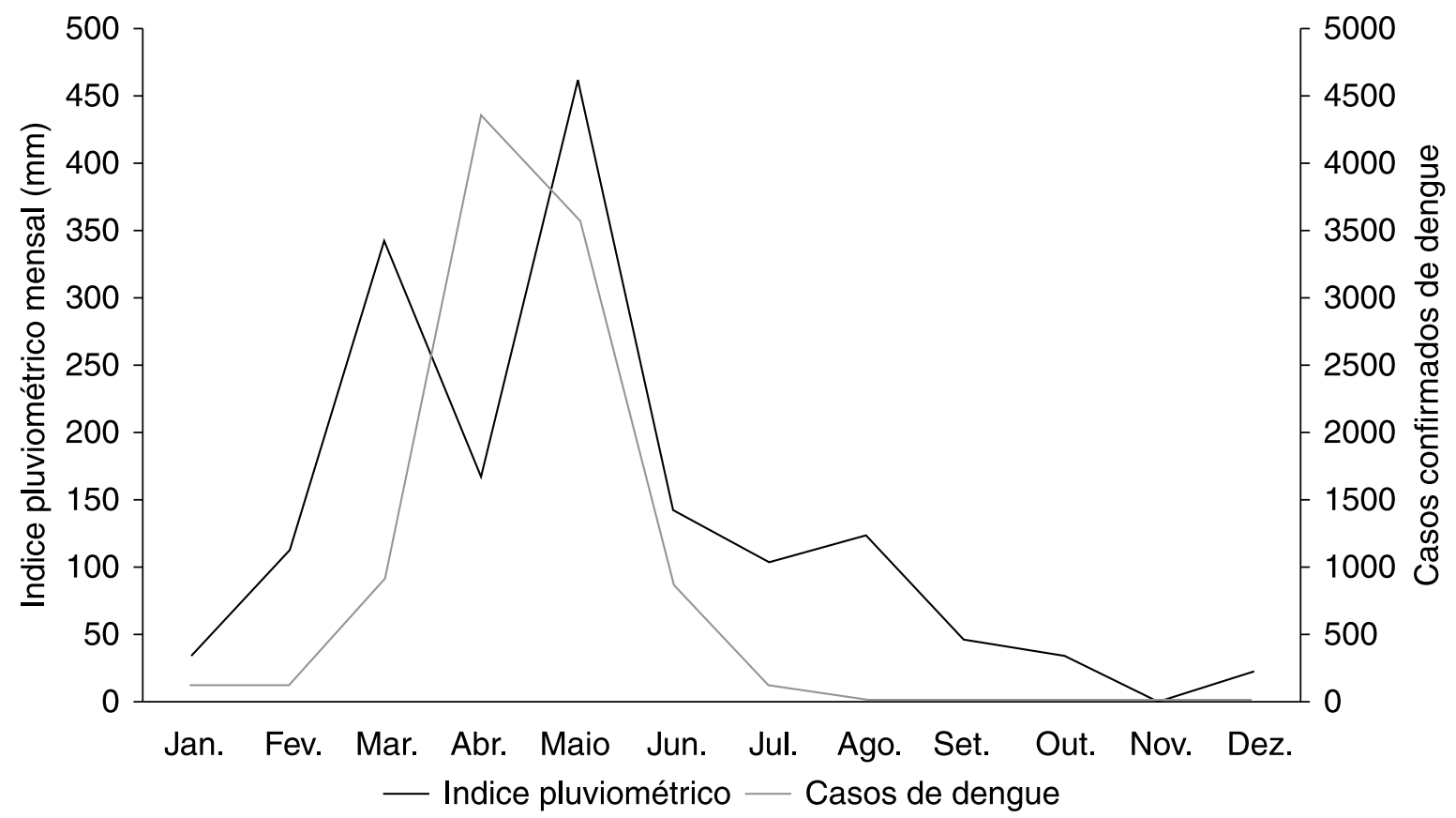

Figura 2. Relação entre o número de casos notificados de dengue e o índice pluviométrico mensal, em mm, no município de Aracaju, Sergipe, 2008. Fonte: SINAN/Centro de Meteorologia da Secretaria de Estado do Meio Ambiente e dos Recursos Hídricos, $(2012)^{14}$.

A faixa etária mais atingida foi a de menores de 15 anos, seguida pelos indivíduos entre 15 a 29 anos. Gonçalves Neto \& Rebelo ${ }^{17}$, em estudo epidemiológico da dengue na cidade de São Luis (MA) no período de 1997 a 2002, observaram que a faixa etária mais acometida foi de 15 a 49 anos, o que representou $72,2 \%$ dos casos $(p<0,05)$, resultado este que difere do presente estudo, pois mais de 37,4\% dos casos estão distribuídos em menores de 15 anos. Já Barbosa et al. ${ }^{16}$, em estudo sobre série temporal no Estado do Rio Grande do Norte (2001 a 2009), observaram que a faixa etária mais acometida foi a de adultos jovens (15-34 anos). Por outro lado, o Ministério da Saúde aponta que a partir de 2008, a doença foi caracterizada por um padrão de migração de gravidade para as crianças ${ }^{8}$.

Avaliando a faixa etária isoladamente, Brito ${ }^{18}$ sintetiza que esta náo pode ser considerada um fator associado a formas mais graves da dengue, podendo apresentar variaçóes entre regiôes, diferentes epidemias e o período estudado. A idade não apresentou influência nos casos de óbito no presente estudo, $\operatorname{com} \mathrm{p}=0,9876$. Porém, comparando-se com as outras faixas etárias, observou-se que as faixas de 45 a 60 anos e maiores de 60 anos apresentaram os maiores coeficientes de mortalidade. A mortalidade elevada a partir dos 45 anos de idade sugere que a presença de outras co-morbidades preexistentes pode ser fator agravante para os casos de DCC, FHD e SCD.

Com relação à cor da pele, observou-se que as fichas apresentavam cor de pele ignorada em $51,7 \%$ dos casos. Vasconcelos ${ }^{19}$, em estudo com dados do SINAN também encontrou uma alta taxa de casos ignorados quanto á variável raça/cor da pele (76,6\%). A raça também foi um indicador negligenciado, nos anos de 2001, 2002 e 2003, o percentual de ignorados ou não preenchidos atingiu 84,6\%, 72,2\% e 41,9\% em Nossa Senhora do Socorro (SE) ${ }^{20}$, município vizinho a Aracaju. A variável raça/cor da pele foi negligenciada, embora se constitua em uma ferramenta de orientação para a análise das desigualdades em saúde, sendo um importante marcador resumo da desigualdade social ${ }^{21}$. Observando apenas os casos de óbito, 76,2\% destes ocorreram em indivíduos pardos ou negros $(\mathrm{p}=0,6053)$.

A regiáo de saúde que apresentou o maior coeficiente de incidência e letalidade foi a quarta região, que possui piores condições de habitação, renda e saneamento do município. Entretanto o resultado difere daquele obtido por Alves et al. ${ }^{22}$, que analisaram a dengue em Aracaju no período de 2001 a 2009, provavelmente, porque os autores trabalharam com os dados agregados no período.

Observa-se na Figura 1 o acompanhamento da epidemia e da sorologia positiva segundo semana epidemiológica. Barros et al. ${ }^{23}$ concluem que mesmo em surtos de dengue, é fundamental que sejam realizados testes específicos para o diagnóstico confirmatório da doença.

Dos casos confirmados, 96,1\% cursaram com DC, em concordância com a tendência brasileira no período epidêmico 9 . A letalidade geral da doença foi de $0,18 \%$. No entanto, se forem considerados os casos notificados graves 
(DCC, FHD e SCD), a letalidade da doença sobe para 4,6\%, acima da média nacional para este ano ${ }^{9}$. Estudos mostram que é possível e factível reduzir a letalidade por dengue hemorrágico a valores em torno de $1 \%$, pois em epidemias em países como Cuba e Venezuela esses valores foram alcançados ${ }^{1}$.

De acordo com a curva dos casos de dengue e os índices pluviométricos (Figura 2), observa-se que o número de casos cresce posteriormente ao período de aumento das chuvas, permanecendo com alta incidência no período compreendido entre a $13^{\mathrm{a}}$ e a $24^{\mathrm{a}}$ semana epidemiológica, período este considerado chuvoso para o município de Aracaju. O decréscimo da incidência a partir da $25^{\mathrm{a}}$ semana retrata a sazonalidade da doença na cidade, decorrente da redução da densidade da população de vetores em função da queda de temperatura e umidade que se registra entre julho e outubro, particularmente na Região Nordeste do Brasil ${ }^{24}$.

Este dado sugere que um dos principais fatores da epidemia de 2008 ocorrida em Aracaju está relacionado ao acúmulo de água limpa e parada das chuvas em recipientes não protegidos e vedados, o que propiciaria a deposição dos ovos do Aedes aegypti, promovendo, assim, a infestação do vetor, principal ponto da campanha no combate a Dengue no Brasil ${ }^{25}$.

Com relaçáo ao resultado do exame, de acordo com as Diretrizes Nacionais para a Prevenção e Controle de Epidemias de Dengue ${ }^{8}$, vigente no período epidêmico, é obrigatório realizar a sorologia de $10 \%$ dos casos suspeitos de Dengue Clássica e em todos os casos graves (DCC, FHD e SCD).

De acordo com a Tabela 2, observa-se que 1.731 casos de dengue foram confirmados laboratorialmente, o que corresponde a $15,4 \%$ do total de casos respeitando, assim, a diretriz. Porém, todos os casos graves obrigatoriamente deveriam ter realizado o exame sorológico e $63,1 \%$ destes não foram confirmados por exame laboratorial, somente por critérios clínico-epidemiológicos. Além disto, foram observadas notificaçôes com sorologia positiva a partir do mês de janeiro (Figura 1), o que sugere a transmissão do agente, podendo ser este mais um instrumento de acompanhamento semanal da doença, além dos seis critérios básicos definidos pelo Ministério da Saúde, em 2011 (incidência atual de casos; incidência de casos nos anos anteriores; índices de infestação pelo Aedes aegypti e sorotipos em circulação; cobertura de abastecimento de água e coleta de lixo; e a densidade populacional) ${ }^{26}$.

A importância da realização do exame sorológico para confirmaçáo dos casos graves de dengue se deve principalmente pela sua letalidade nestes casos. Dos 18 óbitos, todos ocorreram em pacientes com classificação final de DCC ou FHD, condiçóes estas que obrigariam a realização da sorologia em todos eles. No entanto, o exame só foi realizado em 8 dos casos de óbito, obtendo resultado positivo em $75,0 \%$. A proporção de óbito foi maior nos casos em que houve a realização e resultado positivo no exame, sugerindo que a realizaçáo da sorologia é essencial para o diagnóstico dos casos de maior letalidade, porém este resultado não foi estatisticamente significante $(\mathrm{p}=0,1058)$.

Foi observado também, que mesmo casos com resultado positivo ao exame sorológico tiveram fichas que não apresentavam o diagnóstico final como dengue. A sensibilidade para esta relação foi de 99,4\%. Observou-se nos dados do SINAN que, diferentemente do recomendado pelo Ministério da Saúde ${ }^{8}$, muitas vezes a data da sorologia era inferior a seis dias da data do início dos sintomas, o que sugere resultados falso-negativos, pois os anticorpos IgM encontram-se em níveis detectáveis na corrente sanguínea a partir do $5^{\circ}$ ao $6^{\circ}$ dia de evolução da doença ${ }^{24}$.

No presente estudo há limitaçôes quanto à qualidade do registro das fichas de notificação, podendo ter ocasionado possíveis vieses. Porém, este trabalho mostra a grande importância do preenchimento dos dados, pois seus resultados servirão de base para açôes de promoção e prevenção de saúde.

Finalmente, o melhor entendimento epidemiológico da doença permitirá identificar precocemente os pacientes com maior risco de desenvolver as formas graves da doença e possibilitará o desenvolvimento de açóes de combate ao vetor, de aconselhamento da população, buscando a articulação sistemática da vigilância epidemiológica e entomológica com a Atenção Primária a Saúde, integrando suas atividades de maneira a potencializar o trabalho.

\section{Agradecimentos}

Agradecemos ao Dr Marco Prado pela contribuição a este projeto; ao MR Lucas Marques pela sua participaçáo no desenvolvimento do artigo; à Secretaria de Saúde do Estado de Sergipe, principalmente ao Sistema de Informação de Agravos de Notificação, com referência as profissionais Sidney Sá e Ana Carolina Góis. Ao PIBIC/CNPq/UFS pelo apoio ao Projeto de Pesquisa deste trabalho.

\section{Referências}

1. Tauil PL. Aspectos críticos do controle da dengue no Brasil. Cad Saúde Pública. 2002; 18(3): 867-871. http://dx.doi.org/10.1590/ S0102-311X2002000300035

2. Claro LBL, Tomassini HCB, Rosa MLG. Prevenção e controle do dengue: uma revisão de estudos sobre conhecimentos, crenças e práticas da população. Cad Saúde Pública. 2004; 20(6): 1447-1457. http://dx.doi.org/10.1590/S0102-311X2004000600002 
3. Furtado RF, Lima MGA, Bezerra JNS, Silva MGV. Atividade Larvicida de Óleos Essenciais Contra Aedes aegypti L.(Diptera: Culicidae). Neotrop Entomol. 2005; 34(5): 843-847. http://dx.doi.org/10.1590/S1519$566 \times 2005000500018$

4. Costa JGM, Rodrigues FFG, Angélico EC, Silva MR, Mota ML, Santos NKA et al. Estudo químico-biológico dos óleos essenciais de Hyptis martiusii, Lippia sidoides e Syzigium aromaticum frente às larvas do Aedes aegypti. Rev Bras Farmacogn. 2005; 15(4): 304-309.

5. Guzmán MG, Kourí G. Dengue: an update. Lancet. 2002; 2(1): 33-42. http://dx.doi.org/10.1016/S1473-3099(01)00171-2

6. Stephenson JR. Understanding dengue pathogenesis:implications for vaccine design. Bull World Health Organ. 2005; 83(4): 241-300. http:// dx.doi.org/10.1590/S0042-96862005000400016

7. Teixeira MG, Costa MCN, Barreto ML, Mota E. Dengue e febre hemorrágica do dengue no Brasil: que tipo de pesquisas a sua tendência, vigilância e experiências de controle indicam ser necessárias? Cad Saúde Pública. 2005; 5(21): 1307-1315. http:// dx.doi.org/10.1590/S0102-311X2005000500002

8. Brasil. Ministério da Saúde. Secretaria de Vigilância em Saúde. Diretrizes Nacionais para a Prevenção e Controle de Epidemias de Dengue. Brasília; 2009.

9. Brasil. Ministério da Saúde. Secretaria de Vigilância em Saúde. Dengue - Casos da doença no Brasil. [acesso em 2011 Jun 10]. Disponível em: http://portal.saude.gov.br/portal/saude/profissional/ area.cfm?id_area $=1525$

10. Brasil. Ministério da Saúde. Fundação Nacional de Saúde. Programa Nacional de Controle da Dengue - PNCD/Fundação Nacional de Saúde. Brasília; 2002

11. França E, Paula JC, Silva RR, Anunciação LR. Participação da População em Projeto de Controle de Dengue em Belo Horizonte, Minhas Gerais: uma avaliação. Inf Epidemiol SUS. 2002; 11(4): 205-213. http://dx. doi. org/10.5123/S0104-16732002000400003

12. Instituto Brasileiro de Geografia e Estatística - IBGE. Estimativas elaboradas no âmbito do Projeto UNFPA/IBGE (BRA/4/ P31A) - População e Desenvolvimento. Coordenação de População e Indicadores Sociais. 2008 [acesso em 2010 Fev 15]. Disponível em: http://www.ibge.gov.br

13. Sergipe. Secretaria de Estado do Meio Ambiente e dos Recursos Hídricos. Centro de Meteorologia. Sistema de Informação de Agravos de Notificação do estado de Sergipe. SINAN; 2010.

14. Sergipe. Secretaria do Estado do meio ambiente e dos recursos hídricos. Centro de meteorologia da SMARH. Dados diários de precipitação de 2008. 2008 [acesso em 2012 Nov 15]. Disponível em: http://www.semarh.se.gov.br/meteorologia/modules
15. Ribeiro PC, Sousa DC, Araújo TME. Perfil clínico - epidemiológico dos casos suspeitos de Dengue em um bairro da Zona Sul de Teresina. Rev Bras Enferm. 2008; 61(2): 227-32. http://dx.doi.org/10.1590/ S0034-71672008000200013

16. Barbosa IR, Araújo LF, Canindé FC, Araújo RS, Maciel IJ. Epidemiologia do dengue no Estado do Rio Grande do Norte, Brasil, 2000 a 2009. Epidemiol Serv Saúde. 2012; 21(1): 149-157. http://dx.doi. org/10.5123/S1679-49742012000100015

17. Gonçalves Neto VS, Rebelo JMM. Aspectos epidemiológicos do dengue no Município de São Luís, Maranhão, Brasil, 1997-2002. Cad Saúde Pública. 2004; 20(5): 1424-1431. http://dx.doi.org/10.1590/ S0102-311X2004000500039

18. Brito CAA. Dengue em Recife, Pernambuco: padrões clínicos, epidemiológicos, laboratoriais e fatores de risco associados à forma grave da doença. [Dissertação]. Recife: Centro de Pesquisas Aggeu Magalhães, Fundação Oswaldo Cruz; 2007.

19. Vasconcelos SS, Thule LCS, Girianell VR. Incidência das Meningites no Estado do Rio de Janeiro no período de 2000 a 2006. Rev Bras Neurol. 2011; 47(1): 7-14.

20. Cunha LAD, Guedes SAG. Prevalência de esquistossomose mansônica na cidade de Nossa Senhora do Socorro, Sergipe, 2001-2006. Ideias \& Inovação. 2012; 1(1): 41-48.

21. Fiorio NM. Mortalidade por raça/cor em Vitória (ES): análise das informações e das desigualdades em saúde. [Dissertação]. Vitória: Centro de Ciências da Saúde, Universidade Federal do Espirito Santo; 2009.

22. Alves JAB, Santos JR, Mendonça EN, Abud ACF, Nunes MS, Fakhouri $R$, et al. Aspectos epidemiológicos da dengue em Aracaju, Estado de Sergipe, Brasil. Rev Soc Med Trop. 2011; 44(6): 670-673. http://dx.doi. org/10.1590/S0037-86822011000600004

23. Barros LPS, Igawa SES, Jocundo SY, Brito Junior LC. Análise crítica dos achados hematológicos e sorológicos de pacientes com suspeita de Dengue. Rev Bras Hematol Hemoter. 2008; 30(5): 363-366. http:// dx.doi.org/10.1590/S1516-84842008000500007

24. Teixeira MG, Costa MCN, Barreto ML, Barreto FR. Epidemiologia da dengue em Salvador-Bahia, 1995-1999. Rev Soc Bras Med Trop. 2001; 34(3): 269-274. http://dx.doi.org/10.1590/S003786822001000300007

25. Brasil. Ministério da Saúde. Fundação Nacional de Saúde. Manual de dengue - Vigilância epidemiológica e atenção ao doente. 2. ed. Brasília, 1996.

26. Brasil. Ministério da Saúde. Fundação Oswaldo Cruz - Fiocruz. Rede de ações integradas de atenção á saúde no controle da dengue. Risco de dengue. [acesso em 2012 Nov 15] Disponível em: http://www. fiocruz.br/rededengue/cgi/cgilua.exe/sys/start.htm?infoid=37\&sid=9 